\title{
Caractéristiques du peuplement ligneux de deux systèmes d'utilisation des terres dans la région de Kaffrine au Sénégal
}

\author{
Abdou Dione ${ }^{1,2 *}$ Saliou Ngom ${ }^{2}$ Oumar Sarr ${ }^{1}$ Aly Diallo ${ }^{1,3}$ \\ Aliou Guissé ${ }^{1}$
}

\section{Mots-clés}

Plante ligneuse, ressource alimentaire, système agropastoral, peuplement forestier, Sénégal

Submitted: 31 January 2019

Accepted: 9 October 2020

DOI: $10.19182 /$ remvt.36315

\begin{abstract}
Résumé
L'étude a porté sur la caractérisation du peuplement ligneux de deux systèmes d'utilisation des terres (la forêt qui est une mise en défens et les champs) dans le terroir de Ngouye au Sénégal. Elle a été effectuée dans une zone agropastorale qui accueille annuellement plusieurs familles de transhumants. Au moyen d'un échantillonnage par relevés de la végétation ligneuse et d'enquêtes auprès des populations nous avons inventorié 28 espèces appartenant à 25 genres et 15 familles botaniques. Les Fabaceae (sous-familles : Mimosoideae, Caesalpinioideae, Faboideae) ont été les plus représentées, suivies des Combretaceae. Les paramètres de l'étude ont révélé qu'il existait des différences nettes entre les deux systèmes, ce qui montrait leur hétérogénéité, avec par ailleurs une grande stabilité de peuplement en forêt. Le peuplement était généralement très jeune, avec un grand pourcentage $d^{\prime}$ individus dans la première catégorie de diamètre et de hauteur. Les champs avaient la plus forte proportion d'individus adultes mais le taux de régénération dans cette zone était inférieur à celui de la forêt. La richesse spécifique des champs était bien supérieure à celle de la forêt. Sur le plan pastoral les ligneux intervenaient dans l'alimentation du bétail.
\end{abstract}

- Comment citer cet article: Dione A., Ngom S., Sarr O., Diallo A., Guissé A., 2020. Characteristics of the woody stands of two land-use systems in Kaffrine Region, Senegal. Rev. Elev. Med. Vet. Pays Trop., 73 (3): 221-229, doi: 10.19182/remvt.36315

\section{INTRODUCTION}

Au Sahel, l'élevage est la principale activité de la population rurale avec l'agriculture. Il contribue de $5 \%$ à $10 \%$ du produit intérieur brut (PIB), alors que les systèmes pastoraux fournissent plus de $50 \%$ de la production de lait et de viande dans différents pays (FAO, 1999). Le Sénégal est un pays de la zone du Sahel. L'agriculture constitue une activité de production, représentant $70 \%$ de la population. Elle couvre près de $90 \%$ des surfaces emblavées (FAO, 2003). Cependant elle ne contribue que pour $10 \%$ du PIB tout en absorbant en moyenne le dixième des investissements publics (RS/MEF, 2006). A côté de l'agriculture, l'élevage a un apport important sur l'économie du pays ; véritable « épargne sur pattes » qui permet aux familles paysannes de capitaliser les années fastes et de faire face aux épisodes climatiques secs et à la crise alimentaire (Veillard, 2011), il participe à la sécurité alimentaire.

\footnotetext{
1. Laboratoire d'écologie végétale et écohydrologie, FST-UCAD, BP 5005 DakarFann, Sénégal.

2. Laboratoire national d'élevage et de recherche vétérinaire (LNERV), ISRA, Dakar-Fann, Sénégal.

3. Laboratoire d'agroforesterie et d'écologie, UASZ, Ziguinchor, Sénégal.

* Auteur pour la correspondance

Tél. : +221773448115 ; email : dioneabdou10@gmail.com
}

Cependant, cet élevage est caractérisé par la dominance du système extensif (Kiema, 2007) et dépend des pâturages naturels. Ces pâturages contribuent à $90 \%$ dans l'alimentation des herbivores (Zoungrana, 1991). Le système d'alimentation repose principalement sur l'exploitation des ressources fourragères herbacée et ligneuse. Les zones de production ne fournissent généralement pas de sous-produits de l'industrie agricole (principalement des tourteaux d'arachide, de la mélasse). Ces sous-produits sont en partie exportés et vendus à des prix excessivement élevés (Fall et al., 1997). Les cultures fourragères ont été également expérimentées pour lever la contrainte alimentaire dans les systèmes d'élevage mais leur adoption est freinée par les cultures de rente (Ngom et al., 2013).

L'utilisation des ressources naturelles est actuellement la principale stratégie à mettre en place pour améliorer l'état nutritionnel du bétail. Elle devient aujourd'hui la seule alternative qui permettrait de satisfaire les besoins en azote des ruminants dans les élevages traditionnels extensifs et semi-intensifs (Bognounou, 2004). Dans ce cadre, l'importance des ligneux n'est plus à démontrer (Fall et al., 1994).

Le Sénégal est confronté à un énorme déséquilibre écologique. L'explosion démographique actuelle du pays a exacerbé la pauvreté et la dégradation des sols. En effet, les données démographiques élevées ont conduit à davantage d'utilisateurs des terres dans la région. De plus, les pénuries alimentaires peuvent entraîner un surpâturage, perturbant ainsi l'équilibre entre la végétation et le bétail (ACDI, 1985). 
Dans certaines régions du globe aux climats extrêmes, les gens sont plus conscients du changement climatique causé par les émissions mondiales. La zone sahélienne où se trouve le Sénégal en fait partie. Des sécheresses répétées, une distribution insuffisante ou inégale des précipitations temporelles et spatiales, et un épuisement partiel ou complet des eaux souterraines sont les effets du changement climatique au Sénégal. Combinés aux facteurs socioéconomiques de la désertification, ils peuvent entraîner de multiples conséquences, comme une diminution de la fertilité des sols et une érosion accélérée des sols. Le phénomène de désertification au Sahel a des conséquences non seulement environnementales mais également socioéconomiques (GIEC, 2007). Dans ce contexte, il est important d'étudier les ligneux existants pour leur meilleure utilisation par les agropasteurs. C'est pourquoi plusieurs études concernant l'arbre en milieu soudano-sahélien ont étés entreprises dans la zone du bassin arachidier (Ngom, 2001 ; Bakhoum, 2013 ; Sarr et al., 2013c) particulièrement dans la région de Kaffrine. La présente étude est une contribution à la connaissance des ligneux dans la région de Kaffrine. L'objectif global était de caractériser le peuplement ligneux dans le terroir de Ngouye au centre du Sénégal.

\section{MATERIEL ET METHODES}

\section{Site de l'étude}

L'étude a été menée dans la région de Kaffrine qui est entourée des régions de Diourbel, Louga et Matam au nord, de la République de Gambie au sud, de la région de Tambacounda à l'est, et de la région de Kaolack à l'ouest. Kaffrine est située entre $12^{\circ} 06^{\prime}$ et $14^{\circ} 10^{\prime} \mathrm{N}$, et entre $15^{\circ}$ $33^{\prime}$ et $15^{\circ} 53^{\prime} \mathrm{O}$. Sa population est estimée à 566992 habitants (ANSD, 2013). Le site de Ngouye (figure 1), département de Birkelane dans la commune de Ndiognick, a été retenu comme zone d'étude car c'est une zone agropastorale qui accueille annuellement plusieurs familles de transhumants. En effet, il s'agit d'une zone de transition écologique entre les éleveurs du nord du Sahel et du sud du Soudan. En saison sèche, cette zone est la terre d'accueil ou le lieu de passage de nombreux ruminants. Le développement frontalier de l'agriculture exerce une pression énorme sur les ressources ligneuses disponibles (Sarr et al., 2013c).

\section{Climat}

Le climat est de type sahélien au nord et sahélo-soudanien vers le sud avec des précipitations dont l'inégalité dans l'espace et la faiblesse s'accentuent du sud vers le nord (figure 2). La région connait des températures mensuelles moyennes comprises respectivement entre $14,9^{\circ} \mathrm{C}$ (minimales) et $43,1^{\circ} \mathrm{C}$ (maximales) (Station de Koungheul). La température minimale est enregistrée en janvier avec des nuits assez fraîches et des températures souvent inférieures à $15^{\circ} \mathrm{C}$ alors que la température maximale atteint plus de $40{ }^{\circ} \mathrm{C}$ en avril. La température moyenne annuelle est environ de $29,6^{\circ} \mathrm{C}$. Les précipitations sont irrégulières. La pluviométrie moyenne de 1965 à 2013 à la station de référence (Koungheul, Kaffrine) était de 625,2 mm. Ces zones sont caractérisées par une pluviométrie insuffisante depuis 1970, dont la plupart ont connu des années de sécheresse biologique, entraînant des précipitations totales inférieures à la moyenne annuelle (équivalant à 24 années de sécheresse) (figure 3). Il y a généralement deux périodes dans l'année : la période sèche de 7 à 8 mois (octobre à mai) $(\mathrm{P}<2 \mathrm{~T}$, où $\mathrm{P}$ et $\mathrm{T}$ correspondent respectivement aux précipitations moyennes mensuelles et à la température de la zone), et la saison des pluies de 4 à 5 mois (mai à septembre) $(\mathrm{P}>2 \mathrm{~T})$ (figure 4). Les précipitations totales en juillet, août et septembre sont de $82,12 \%$, soit une période biologiquement humide ; août est le mois le plus pluvieux (30,94\%).

\section{Reliefs et sols}

Les reliefs sont généralement plats et présentent une légère pente descendant du nord au sud. Le sol rencontré est divisé en trois types
(SRSD Kaffrine, 2013) : a) sols ferrugineux tropicaux (avec des variations) pour cultiver des arachides et du mil, et sableux avec une bonne capacité de production agricole ; b) sols hydromorphes représentant les basses terres et les rivières, dispersés dans la zone, généralement argileux, également appelés « deck » avec une variante « dior»; et c) sols halomorphes caractéristiques des milieux salés ou tannes se trouvant surtout dans la section de Birkelane ; le matériau est généralement de la boue, sinon du limon.

\section{Hydrographie}

La région possède de riches ressources et potentiels hydrogéologiques et hydrologiques. Les ressources en eau de surface comprennent de nombreuses rivières et des marigots temporaires ou des bas-fonds, en particulier les vallées fossiles du Saloum, du Baobolong. La nappe phréatique de la région de Kafflin se situe dans les structures géologiques suivantes : terminal continental, Eocène (Luttian), Paléocène et Maastricht (SRSD Kaffrine, 2013).

\section{Végétation}

La végétation de cette zone est très diversifiée. Il existe plusieurs types de formations forestières du nord au sud. Au nord, il y a une

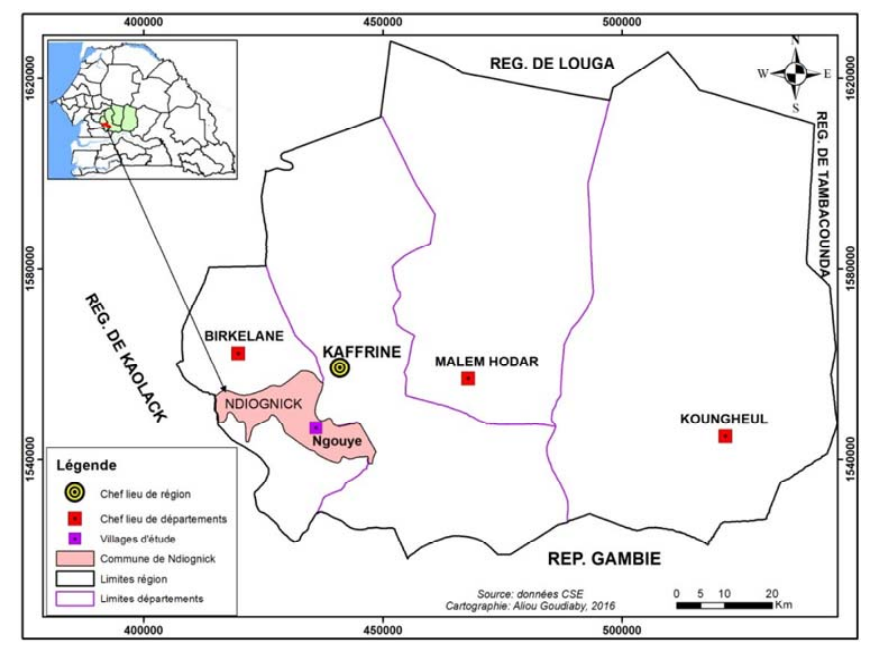

Figure 1 : situation géographique du terroir de Ngouye, commune de Ndiognick, Sénégal. Source : CSE ; cartographie : A. Goudiaby, 2016.

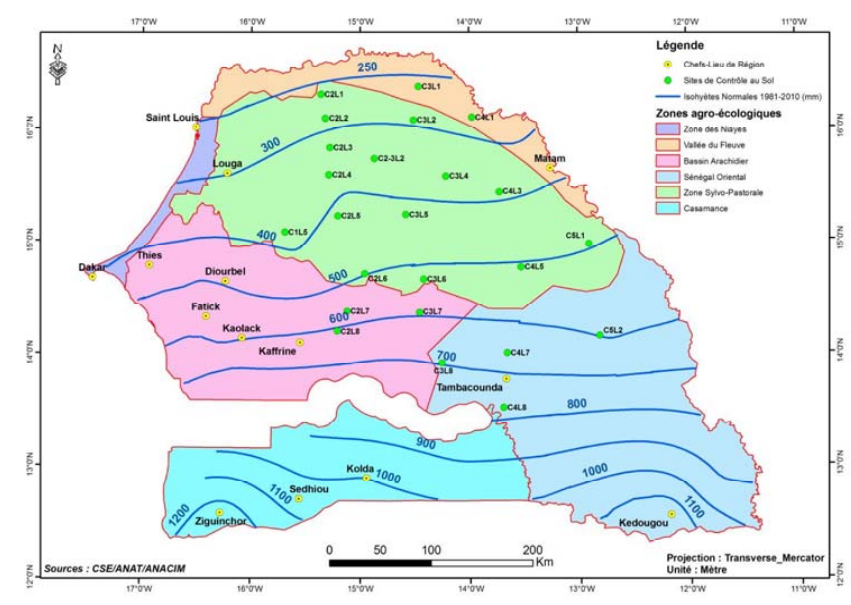

Figure 2 : carte des zones agroécologiques du Sénégal, avec les sites de contrôle au sol et les isohyètes de la moyenne 19812010. Source : CSE/ANAT/ANACIM. 


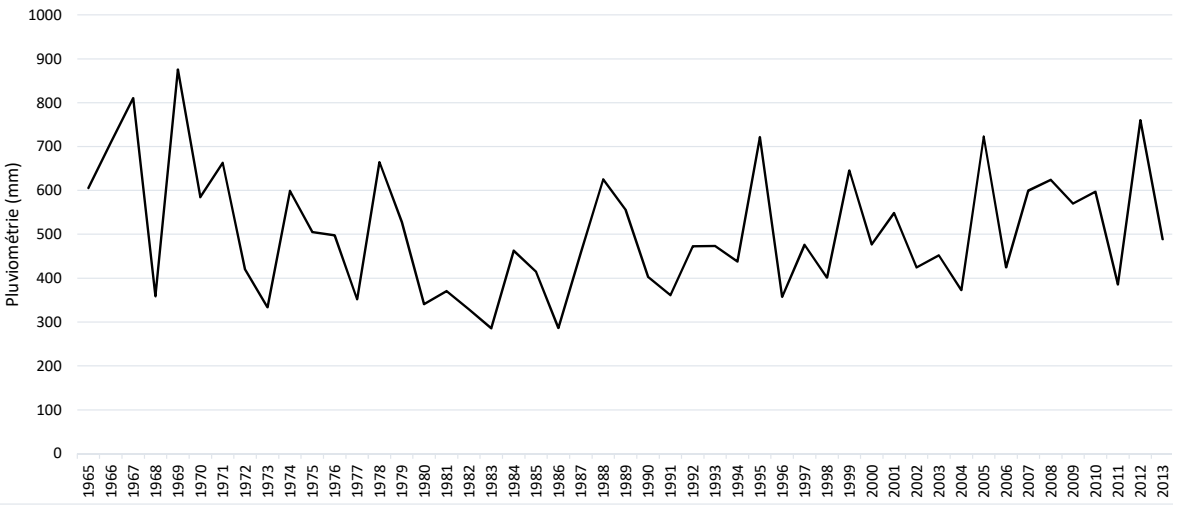

Figure 3 : variabilité interannuelle de la pluviométrie de la station de Kaffrine, Sénégal (ANACIM).

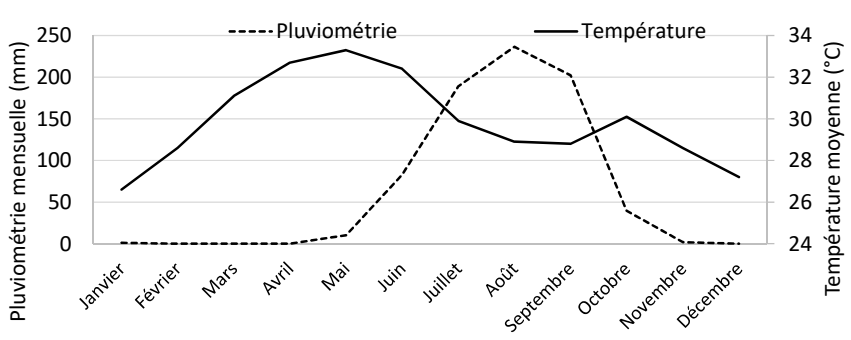

Figure 4 : diagramme ombrothermique de la région de Kaffrine, Sénégal ; pluviométrie moyenne mensuelle et température moyenne mensuelle (minimale et maximale) de 2000 à 2008 (ANACIM).

savane arbustive, qui se caractérise par un écosystème qui rassemble des espèces forestières typiques du Sahel : Combretum sp., Balanites aegyptiaca, Lannea acida, Bauhinia rufescens, Adansonia digitata, Anogeissus leiocarpus, etc. Au sud, la prairie boisée présente les espèces les plus communes suivantes et une grande variété de flores : Cordyla pinnata, Pterocarpus erinaceus, Daniellia oliveri, Parkia biglobosa, Tamarindus indica, Prosopis africana, Sterculia setigera, Parinari macrophylla. En effet, la zone de Kaffrine compte 11 forêts classées et deux réserves sylvopastorales, couvrant une superficie de 241850 hectares (ANSD/SRSD Kaffrine, 2013).

\section{Activités de la population}

La population est principalement engagée dans la production agricole d'arachides et de mil. Depuis 2003, la culture du maïs et des haricots (niébé) est encouragée pour réaliser une diversification alimentaire. L'élevage concerne les bovins, les ovins et les caprins. Les chevaux et les ânes sont utilisés pour l'agriculture, et le transport urbain des marchandises et des produits agricoles. L'extraction du bois de feu et des produits forestiers non ligneux est réalisée principalement par les femmes et les enfants. Ces produits sont vendus en bordure de route et de marchés de fruits locaux (tamarin, jujube, etc.) (Mohamed et al., 2008).

\section{Matériel de terrain}

Pour mener cette étude le matériel suivant a été utilisé : un géopositionnement par satellite (GPS) de marque Garmin pour géoréférencer les points d'échantillonnage sur le terrain et faciliter le tracé des placettes ; des piquets en fer pour la délimitation de chaque côté d'une parcelle ; un penta décamètre pour la délimitation des placettes, la mesure de la distance entre les arbres et la mesure du diamètre du houppier est-ouest et nord-sud de chaque individu ; un ruban de 1,5 $\mathrm{m}$ de long pour mesurer la circonférence à $0,30 \mathrm{~m}$ ou à $1,30 \mathrm{~m}$ du sol de chaque individu recensé et la hauteur de la première branche vivante à partir du sol ; un BlumLeiss pour obtenir la hauteur des arbres depuis le sol jusqu'à la cime ; des fiches de relevés de végétation, des crayons et autres marqueurs pour l'enregistrement des données de terrain ; enfin, la flore du Sénégal de Berhaut (1967) et l'ouvrage des noms vernaculaires des ligneux de Guerin (1984) pour l'identification des espèces sur le terrain.

\section{Méthodes}

Pour caractériser le peuplement ligneux, la méthode de relevé de la végétation (Gounot, 1969) a été utilisée dans les deux systèmes d'utilisation des terres. Quarante relevés ont été effectués dont 20 placettes dans la forêt (même en pleine forêt) et 20 placettes dans les champs. La superficie d'échantillonnage de $2500 \mathrm{~m}^{2}$ (50 m x 50 m) par relevé correspond à l'aire minimale proposée par Boudet (1984) pour l'étude de la végétation ligneuse sahélienne (figure 5).

A l'intérieur de chaque placette, pour apprécier le potentiel de régénération tous les individus dont la circonférence à $30 \mathrm{~cm}$ du sol était supérieure ou égale à $10 \mathrm{~cm}$ ont été recensés et identifiés. Pour chaque individu rencontré, des mensurations ont été effectuées. Les mesures ont porté sur a) la hauteur totale depuis le sol jusqu'à la cime pour établir la structure verticale et horizontale du peuplement, b) la distance entre les arbres par la méthode du plus proche individu pour apprécier la distribution des individus et calculer la densité théorique, c) le diamètre du houppier dans les deux directions est-ouest et nordsud pour évaluer le couvert aérien ; et d) la circonférence à $30 \mathrm{~cm} \mathrm{du}$ sol pour estimer la surface terrière. La dénomination des espèces a été effectuée (Berhaut, 1967 ; Guerin, 1984).

\section{Traitement des données}

Les données ont été traitées avec le tableur Excel. Ainsi nous avons établi une liste floristique et calculé la fréquence de présence des

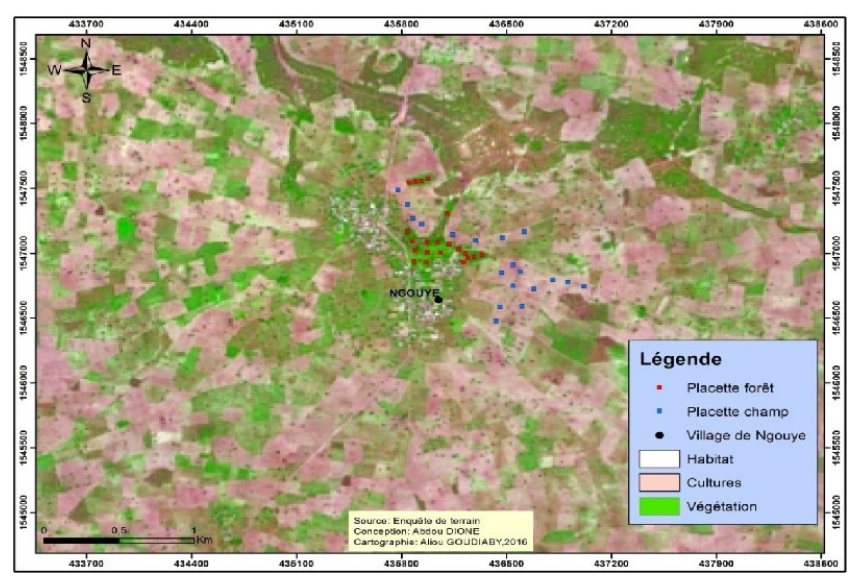

Figure 5 : localisation des placettes d'études dans le terroir de Ngouye, Sénégal. 
espèces, la densité, la surface terrière, la surface de la couronne, le taux et l'importance spécifique de régénération, la structure du peuplement de même que quelques paramètres.

La fréquence renseigne sur la distribution d'une espèce dans les stations. Elle est déterminée par la relation $F=\frac{N i}{N r} \times 100$ avec $N i$ l'effectif total d'une population $i$ et $\mathrm{Nr}$ l'effectif total du peuplement (Roberts-Pichette et Gillespie, 2002).

La densité d'une espèce correspond au nombre de pieds appartenant à l'espèce par unité de surface à l'hectare (individus/ha). Elle est déterminée des deux manières suivantes :

- la densité réelle ou observée $(D o b)$ correspond au nombre réel d'arbres dans la parcelle, ramené à l'hectare. Elle est donnée par le rapport entre l'effectif total de l'espèce dans l'échantillon et la surface de cet échantillon, $D o b=\frac{N i}{S}$ avec $N i$ l'effectif total de l'espèce $i$ dans l'échantillon considéré, et $S$ la surface (ha) de l'échantillon ;

- la densité théorique (Dth), la plus utilisée, est le rapport d'un hectare sur le carré de la distance moyenne entre les arbres. Elle a été obtenue par la formule $D t h=\frac{10000}{(D m)^{2}}$ avec $D m$ la distance moyenne (m) entre les arbres.

Le recouvrement du peuplement ligneux a été apprécié par la détermination de la surface terrière et du couvert aérien. La surface terrière $(S t)$, exprimée en mètres carrés par hectare, est la surface occupée par les sections des fûts à $30 \mathrm{~cm}$ du sol. La surface terrière d'une espèce correspond à la somme des surfaces terrières de tous ses individus. Ainsi, pour un peuplement, c'est la somme des surfaces terrières de toutes les populations. Elle dépend donc de la densité et de la grosseur des pieds (Roberts-Pichette et Gillespie, 2002). Elle se calcule à l'aide de la formule $S t=\sum \frac{C^{2}}{4 \pi}$ avec $C$ la circonférence à $30 \mathrm{~cm}$ du sol des individus mesurés.

Le couvert aérien correspond à la proportion d'un échantillon occupé au sol par la couronne d'un végétal (Roberts-Pichette et Gillespie, 2002). Il est exprimé en mètres carrés par hectare. Le couvert aérien d'une espèce est égal à la somme des surfaces de la couronne de tous les individus de la population. Cette surface de la couronne $(S c)$ est obtenue à l'aide du diamètre moyen du houppier de l'arbre assimilable à un cercle par projection sur le sol. Selon Rondeux (1993), elle est obtenue par la formule $S c=\sum \frac{\pi D^{2}}{4}$ avec $D$ la moyenne des diamètres est-ouest et nord-sud.

Les capacités de régénération des sites ont été appréciées par le calcul du taux de régénération du peuplement (TRP) et de l'importance spécifique de régénération (ISR). Le TRP est donné par le rapport en pourcentage entre l'effectif total des jeunes plants et l'effectif total du peuplement (Poupon, 1980) : TRP $=\sum \frac{\text { Effectif total des jeunes plants }}{\text { Effectif total du peuplement }} \times 100$

L'effectif total du peuplement regroupe aussi bien les jeunes plants que les plants adultes. L'ISR est obtenue à partir du rapport en pourcentage entre l'effectif d'une espèce et l'effectif total de tous les jeunes plants dénombrés (Akpo et Grouzis, 1996) : ISR = $\sum \frac{\text { Effectif des jeunes plants d'une espèce }}{\text { Effectif total des jeunes plants dénombrés }} \times 100$

\section{RESULTATS}

\section{Cortège floristique}

La liste de végétation permet de dresser une liste d'espèces végétales dans les champs et les forêts. La flore ligneuse inventoriée dans la zone d'étude était riche de 28 espèces appartenant à 25 genres et 15 familles (tableau I). A l'exception des Anarcadiaceae ( 2 genres et 2 espèces), des Combretaceae (4 genres et 5 espèces) et des Fabaceae avec les trois sous-genres Caesalpinioideae (4 genres et 4 espèces), Mimosoideae ( 3 genres et 4 espèces) et Faboideae ( 2 genres et 2 espèces), les familles étaient représentées par une seule espèce. Les
Tableau I

Espèces floristiques recensées dans le terroir de Ngouye au Sénégal

\begin{tabular}{|c|c|c|c|}
\hline Famille & Forêt & Champ & Espèce \\
\hline \multirow[t]{2}{*}{ Anacardiaceae } & & + & Anacardium occidentale L. \\
\hline & & + & $\begin{array}{l}\text { Ozoroa insignis Del.(syn. Heeria } \\
\text { insignis (Del.) Kuntze) }\end{array}$ \\
\hline Arecaceae & & + & Borassus aethiopium, Mart. \\
\hline Balanitaceae & + & + & Balanites aegyptiaca (L.) Del. \\
\hline Bignoniaceae & & + & $\begin{array}{l}\text { Stereospermum kunthianum } \\
\text { Cham. }\end{array}$ \\
\hline Bombacaceae & & + & Adansonia digitata L. \\
\hline \multirow[t]{4}{*}{ Caesalpiniaceae } & + & & Cassia sieberiana DC. \\
\hline & & + & $\begin{array}{l}\text { Cordyla pinnata (Lepr. ex A. } \\
\text { Rich.) Milne-Redh. }\end{array}$ \\
\hline & + & + & $\begin{array}{l}\text { Piliostigma reticulatum (DC.) } \\
\text { Hochst. }\end{array}$ \\
\hline & & + & Tamarindus indica L. \\
\hline \multirow[t]{5}{*}{ Combretaceae } & + & + & $\begin{array}{l}\text { Anogeissus leiocarpus (DC.) } \\
\text { Guill. et Perr. }\end{array}$ \\
\hline & + & + & $\begin{array}{l}\text { Combretum glutinosum Perr. } \\
\text { ex DC. }\end{array}$ \\
\hline & + & + & Guiera senegalensis J.F. Gmel \\
\hline & & + & $\begin{array}{l}\text { Terminalia avicennioides Guill. } \\
\text { et Perr. }\end{array}$ \\
\hline & & + & $\begin{array}{l}\text { Terminalia macroptera Guill. } \\
\text { et Perr. }\end{array}$ \\
\hline Ebenaceae & & + & $\begin{array}{l}\text { Diospyros mespiliformis Hochst. } \\
\text { ex DC. }\end{array}$ \\
\hline \multirow{4}{*}{$\begin{array}{l}\text { Fabaceae } \\
\text { (sous-famille } \\
\text { Caesalpinioideae) }\end{array}$} & + & & Cassia sieberiana DC. \\
\hline & & + & $\begin{array}{l}\text { Cordyla pinnata (Lepr. ex A. } \\
\text { Rich.) Milne-Redh. }\end{array}$ \\
\hline & + & + & $\begin{array}{l}\text { Piliostigma reticulatum (DC.) } \\
\text { Hochst. }\end{array}$ \\
\hline & & + & Tamarindus indica L. \\
\hline \multirow[t]{2}{*}{$\begin{array}{l}\text { Fabaceae } \\
\text { (sous-famille } \\
\text { Faboideae) }\end{array}$} & + & & $\begin{array}{l}\text { Senegalia ataxacantha (DC.) } \\
\text { Kyal. \& Boatwr. (syn. Acacia } \\
\text { ataxacantha DC.) }\end{array}$ \\
\hline & & + & $\begin{array}{l}\text { Dichrostachys cinerea (L.) Wight } \\
\text { et Arn. }\end{array}$ \\
\hline \multirow[t]{4}{*}{$\begin{array}{l}\text { Fabaceae } \\
\text { (sous-famille } \\
\text { Mimosoideae) }\end{array}$} & & + & $\begin{array}{l}\text { Vachellia nilotica (L.) P.J.H. } \\
\text { Hurter \& Mabb. (syn. Acacia } \\
\text { nilotica (L.) Willd. ex Del.) }\end{array}$ \\
\hline & + & & $\begin{array}{l}\text { Vachellia seyal (Delile) P.J.H. } \\
\text { Hurter (syn. Acacia seyal Del.) }\end{array}$ \\
\hline & & + & Faidherbia albida (Del.) A. Chev. \\
\hline & & + & $\begin{array}{l}\text { Prosopis africana (Guill. \& Perr.) } \\
\text { Taub. }\end{array}$ \\
\hline Meliaceae & + & + & Azadirachta indica A. Juss. \\
\hline Moraceae & & + & $\begin{array}{l}\text { Ficus sur Forssk. } \\
\text { (syn. Ficus capensis Thunb.) }\end{array}$ \\
\hline Myrtaceae & & + & Eucalyptus alba Reinw. ex Blume \\
\hline Polygalaceae & & + & $\begin{array}{l}\text { Securidaca longipedunculata } \\
\text { Fresen. }\end{array}$ \\
\hline Rhamnaceae & & + & Ziziphus mauritiana Lam. \\
\hline Sterculiaceae & & + & Sterculia setigera Del. \\
\hline
\end{tabular}


espèces communes (champs et forêt) étaient Balanites aegyptiaca, Piliostigma reticulatum, Anogeissus leiocarpus, Combretum glutinosum, Guiera senegalensis et Azadirachta indica.

\section{Fréquence de chaque espèce rencontrée}

Au total, 676 individus ont été répertoriés pour toutes les placettes, soit 566 individus pour la forêt et 110 individus pour les champs (tableau II). Au niveau de la forêt, Combretum glutinosum a présenté la fréquence la plus élevée (56,00 \%), suivie de Guiera senegalensis $(39,75 \%)$. Les autres espèces faisaient moins de $2 \%$. Au niveau des champs, Eucalyptus alba (22,72\%) a été plus fréquente que Cordyla pinnata (21,18\%), venait ensuite Adansonia digitata (18,18\%). Les autres espèces faisaient moins de $8 \%$. Les espèces dont les fréquences étaient plus élevées au niveau de la forêt avaient des fréquences nulles au niveau des champs à l'exception de Combretum glutinosum qui a chuté à $3,63 \%$. Au niveau des champs les trois espèces dont la fréquence était la plus représentative n'ont pas été présentes dans la forêt (Eucalyptus alba, Cordyla pinnata et Adansonia digitata).

\section{Recouvrement}

Le recouvrement basal a été de 34,68 m²/ha pour la forêt et de 77,80 $\mathrm{m}^{2} /$ ha pour les champs (tableau III). Au niveau de la forêt, Combretum glutinosum dominait avec $32,31 \mathrm{~m}^{2} / \mathrm{ha}$. Guiera senegalensis venait ensuite avec 1,22 $\mathrm{m}^{2} / \mathrm{ha}$. Toutes les autres espèces ont eu des recouvrements inférieurs à $1 \mathrm{~m}^{2} / \mathrm{ha}$. Au niveau des champs, Adansonia digitata $\left(49,23 \mathrm{~m}^{2} / \mathrm{ha}\right)$ dominait suivis de Cordyla pinnata $(9,26 \mathrm{~m} / \mathrm{ha})$ et Ficus $\operatorname{sur}\left(7,65 \mathrm{~m}^{2} / \mathrm{ha}\right)$. Toutes les autres espèces ont eu des recouvrements inférieurs à $4 \mathrm{~m}^{2} /$ ha. Le couvert aérien a été de $5980,11 \mathrm{~m}^{2} /$ ha pour la forêt et de 7253,86 m²/ha pour les champs (tableau III). Au niveau de la forêt, Combretum glutinosum recouvrait plus de la moitié de la surface avec 3248,94 m²/ha, suivi de Guiera senegalensis $\left(1972,99 \mathrm{~m}^{2} / \mathrm{ha}\right)$ et

\section{Tableau III}

Quelques paramètres écologiques de la végétation ligneuse dans le terroir de Ngouye au Sénégal

$\begin{array}{lcc}\text { Paramètre } & \text { Forêt } & \text { Champ } \\ \text { Richesse spécifique } & 9 & 25 \\ \text { Recouvrement aérien (m²/ha) } & 5980 & 7254 \\ \text { Recouvrement basal (m²/ha) } & 34,7 & 77,8 \\ \text { Régénérations (\%) } & 98,4 & 76,3 \\ \text { Distance moyenne (m) } & 7,7 & 30,1 \\ \text { Densités réelles (ind./ha) } & 113,2 & 22.0 \\ \text { Densités théoriques (ind./ha) } & 170,4 & 11,1\end{array}$

\section{Tableau II}

Densité relative, surface terrière et couvert aérien des espèces dans le terroir de Ngouye au Sénégal

\begin{tabular}{|c|c|c|c|c|c|c|}
\hline & \multicolumn{2}{|c|}{ Densité relative (\%) } & \multicolumn{2}{|c|}{ Surface terrière $\left(\mathrm{m}^{2} / \mathrm{ha}\right)$} & \multicolumn{2}{|c|}{ Couvert aérien $\left(\mathrm{m}^{2} / \mathrm{ha}\right)$} \\
\hline & Champs & Forêt & Champs & Forêt & Champs & Forêt \\
\hline Adansonia digitata & 18,2 & & 49,2 & & 1443,6 & \\
\hline Anacardium occidentale & 7,3 & & 1,3 & & 544,5 & \\
\hline Anogeissus leiocarpus & 1,8 & 0,4 & 3,5 & 0,4 & 262,8 & 99,1 \\
\hline Azadirachta indica & 4,5 & 1,2 & 0,6 & 0,6 & 131,4 & 84,3 \\
\hline Balanites aegyptiaca & 0,9 & 0,2 & $<0,1$ & $<0,1$ & 27,3 & 4,6 \\
\hline Borassus aethiopium & 6,4 & & 3,2 & & 111,2 & \\
\hline Cassia sieberiana & & 0,4 & & 0,1 & & 56,4 \\
\hline Combretum glutinosum & 3,6 & 56,0 & 0,1 & 32,3 & 32,7 & 3248,9 \\
\hline Cordyla pinnata & 21,2 & & 9,3 & & 2816,7 & \\
\hline Dichrostachys cinerea & 0,9 & & $<0,1$ & & 20,4 & \\
\hline Diospyros mespiliformis & 0,9 & & $<0,1$ & & 22,6 & \\
\hline Eucalyptus alba & 22,7 & & 0,4 & & 368,8 & \\
\hline Faidherbia albida & 0,9 & & $<0,1$ & & 10,7 & \\
\hline Ficus sur & 1,8 & & 7,7 & & 549,1 & \\
\hline Guiera senegalensis & 0,0 & 39,8 & 0,0 & 1,2 & 0,0 & 1973,0 \\
\hline Ozoroa insignis & 0,0 & & 0,0 & & 0,0 & \\
\hline Piliostigma reticulatum & 0,0 & 0,0 & 0,0 & 0,0 & 0,0 & 0,0 \\
\hline Prosopis africana & 0,9 & & 0,1 & & 25,5 & \\
\hline Securidaca longipedunculata & 0,0 & & 0,0 & & 0,0 & \\
\hline Senegalia ataxacantha & & 0,2 & & $<0,1$ & & 2,4 \\
\hline Sterculia setigera & 0,9 & & 0,9 & & 148,5 & \\
\hline Sterospermum kunthianum & 0,0 & & 0,0 & & 0,0 & \\
\hline Tamarindus indica & 4,5 & & 1,7 & & 696,1 & \\
\hline Terminalia avicennoides & 0,0 & & 0,0 & & 0,0 & \\
\hline Terminalia macroptera & 0,0 & & 0,0 & & 0,0 & \\
\hline Vachellia nilotica & 0,9 & & $<0,1$ & & 31,4 & \\
\hline Vachellia seyal & & 1,9 & & 0,1 & & 511,3 \\
\hline Ziziphus mauritiana & 0,9 & & $<0,1$ & & 10,8 & \\
\hline
\end{tabular}


Vachellia seyal $(511,29 \mathrm{~m} / \mathrm{ha})$. Au niveau des champs, Cordyla pinnata recouvrait une surface plus importante avec $2816,71 \mathrm{~m}^{2} / \mathrm{ha}$, suivi de Adansonia digitata (1443,58 $\left.\mathrm{m}^{2} / \mathrm{ha}\right)$. Le tableau III détaille le couvert aérien de chaque espèce dans les champs et la forêt.

\section{Paramètres de diversité}

Les champs ont été nettement plus riches en espèces avec 25 espèces contre 9 dans la forêt (tableau III). Il en était de même pour le couvert aérien et le recouvrement basal. Cependant, la forêt s'est mieux régénérée avec un taux de régénération de $98,4 \%$ alors que celui des champs a été de 76,34\%. La densité réelle la plus élevée a été notée dans la forêt avec 113,2 individus/ha contre 22 ind./ha dans les champs. La distance moyenne entre les individus les plus proches a été de 7,66 m dans la forêt et de 0,06 m dans les champs soit des densités théoriques respectives de 170,42 ind./ha et 11,06 ind./ha. Au niveau de la forêt la densité réelle a été inférieure à la densité théorique, au niveau des champs c'était le contraire.

\section{Structure du peuplement selon la circonférence}

La figure 6 présente la distribution selon les classes de circonférences des individus au niveau de la forêt et au niveau des champs. Elle s'apparente à une distribution asymétrique droite avec un paramètre de forme compris entre 1 et 3,6. Les sujets dont la circonférence était comprise entre 10 et $40 \mathrm{~cm}$ étaient les plus nombreux. La classe des circonférences comprises entre 10 et $30 \mathrm{~cm}$ comportait le plus grand nombre d'individus. Cette figure a montré que la structure en circonférence des individus au niveau des champs présentait une distribution en « J inversé » avec un paramètre de forme inférieur à un. L'essentiel des individus avaient une circonférence comprise entre 50 et $250 \mathrm{~cm}$.

\section{Structure du peuplement selon la hauteur}

La distribution selon la hauteur des individus au niveau de la forêt et
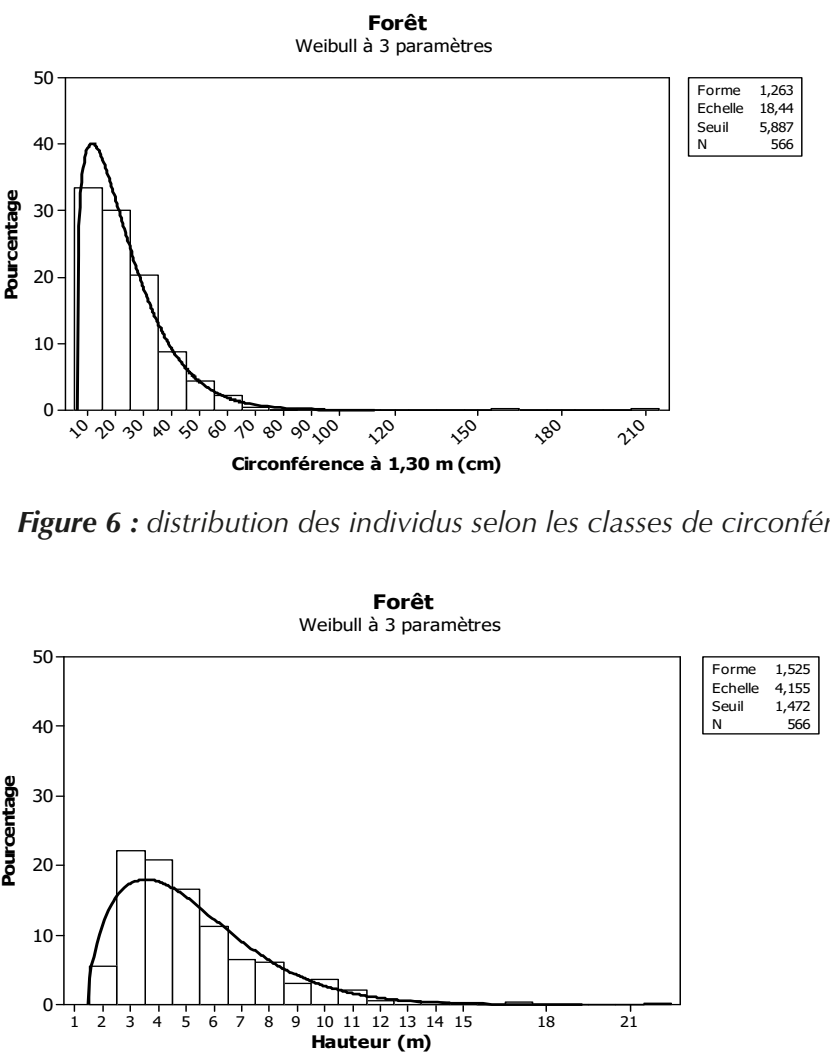

population était constituée d'individus à hauteur inférieure à $7 \mathrm{~m}$. Les individus dont la taille était comprise entre 3 et $4 \mathrm{~m}$ étaient plus nombreux. Toutefois, la strate arborescente (individus dont la hauteur excédait $7 \mathrm{~m}$ ) était peu représentée. Au niveau des champs, l'inverse a été observé avec une prédominance des individus de grande taille. Les individus les plus représentés avaient une taille comprise entre 7,5 et $21 \mathrm{~m}$.

\section{Capacité de régénération}

Au total, 35557 individus jeunes représentant 16 espèces ont été inventoriés et consignés dans la figure 8 . Au niveau de la forêt où le peuplement se régénérait à $98,4 \%$, nous avons noté le plus grand nombre avec 35201 régénérations (20 501 individus pour Combretum glutinosum et 14300 pour Guiera senegalensis) soit des importances spécifiques de régénération de 58,23\% et 40,52\%. Les autres espèces avaient un taux inférieur à $1 \%$. Les champs comptaient 356 rejets. Les jeunes plantes représentaient 76,34 \% et les plus abondantes parmi ces dernières étaient Guiera senegalensis (34,83\%), Piliostigma reticulatum (22,74 \%) et Ozoroa insignis (11,58 \%). Les autres espèces avaient un taux inférieur à $5 \%$.

\section{DISCUSSION}

L'étude a révélé que le cortège floristique de l'ensemble champs et forêt était constitué de 28 espèces ligneuses réparties entre 25 genres et 15 familles. Sarr et al. (2013c), qui ont travaillé sur l'importance des ligneux fourragers dans un système agropastoral à Kaffrine, rapportent 51 espèces appartenant à 41 genres et 22 familles. Ces différences pourraient s'expliquer, d'une part, par les surfaces échantillonnées et le niveau d'anthropisation. D'autre part, elles pourraient provenir de la répartition de la pluviométrie, de la nature du sol et de la situation topographique, qui selon Breman et Kessler (1995) sont des facteurs auxquels la composition floristique des pâturages est étroitement liée.

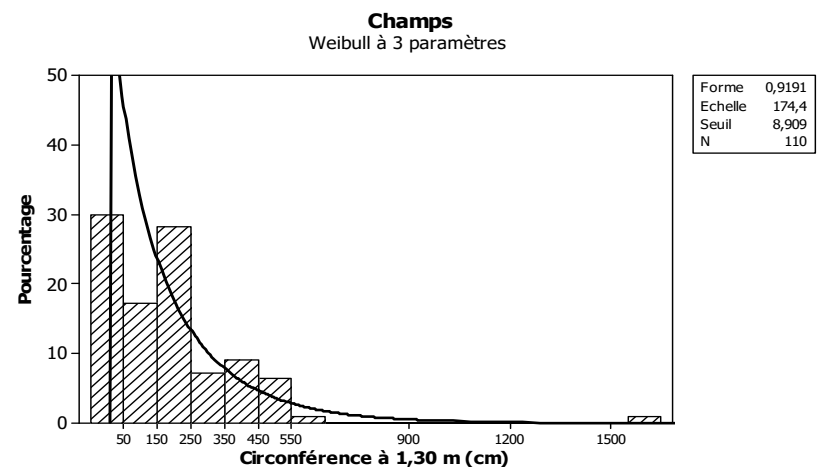

Figure 6 : distribution des individus selon les classes de circonférences dans la forêt et dans les champs, terroir de Ngouye, Sénégal.

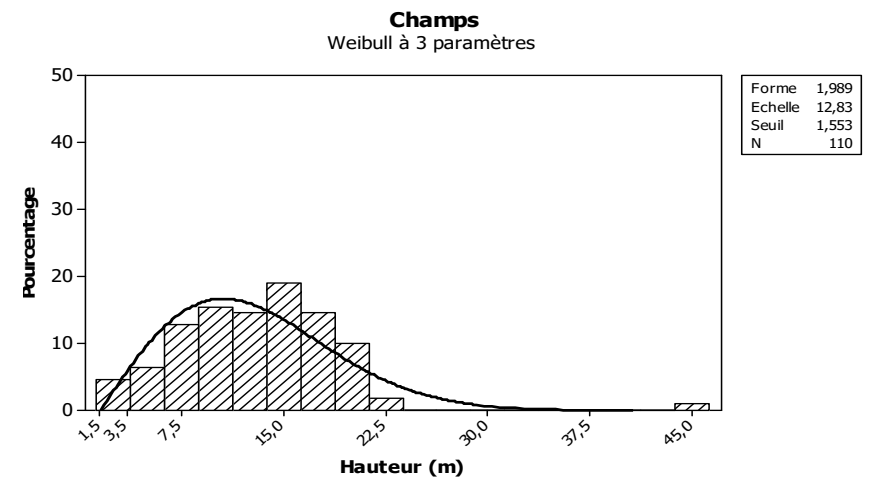

Figure 7 : distribution des individus selon les classes de hauteurs dans la forêt et dans les champs, terroir de Ngouye, Sénégal. 


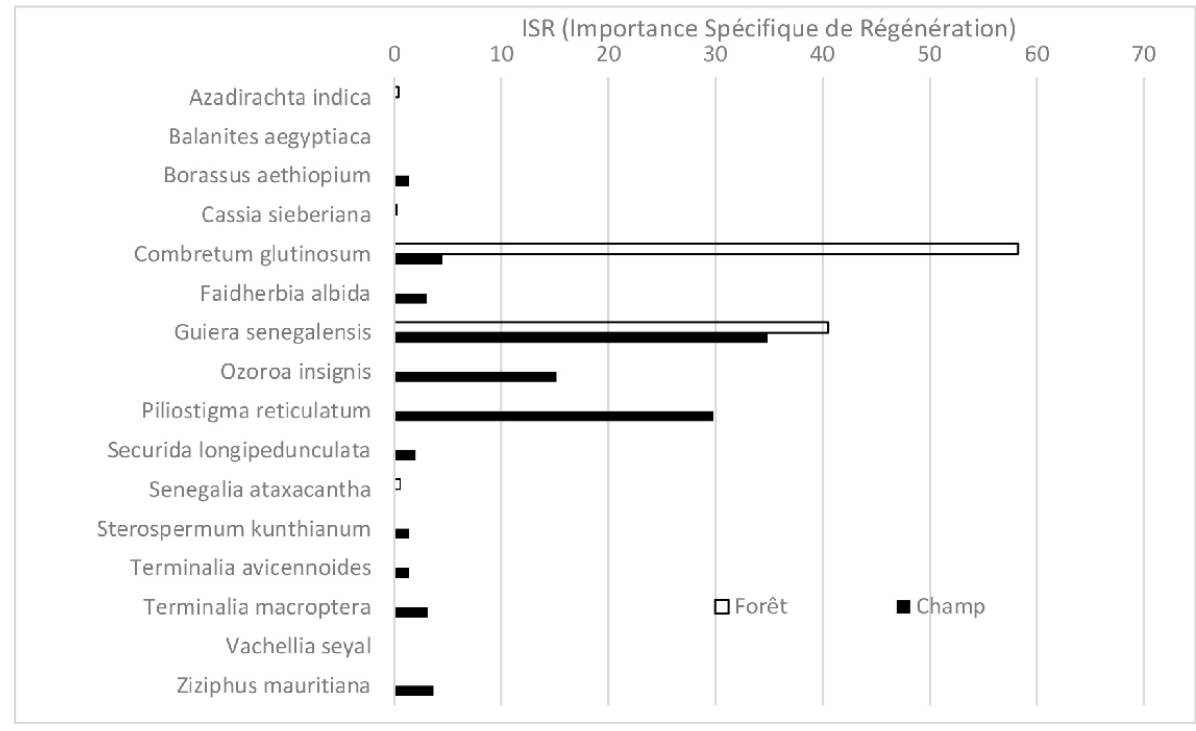

Figure 8 : importances spécifiques de la régénération de chaque espèce suivant le site, terroir de Ngouye, Sénégal.
Les analyses ont permis de distinguer deux groupes d'espèces selon leurs fréquences dans les différentes unités de production. Les espèces très fréquentes (dominantes) ont été Combretum glutinosum et Guiera senegalensis dans la forêt, et Eucalyptus alba, Cordyla pinnata et Adansonia digitata dans les champs.

Les champs ont eu une richesse spécifique ligneuse plus élevée que la forêt et ont regroupé également les six espèces ligneuses les plus importantes retenues par les producteurs. La forêt a totalisé moins d'espèces. Les méthodes d'aménagement des terres agricoles étant considérées comme destructrices pour la biodiversité ligneuse, ce résultat semble paradoxal. Cela peut s'expliquer par le reboisement de certaines espèces, comme Eucalyptus alba, Azadirachta indica, Anacardium occidentale. Cela augmente la diversité des terres cultivées, ce qui montre que les populations rurales comprennent bien le rôle écologique des arbres ligneux. Un constat similaire a été noté par Sarr et al. (2014) dans des agrosystèmes de Koungheul. La famille la plus représentative dans notre étude était celle des Fabaceae avec les sous-familles Caesalpinioideae, Mimosoideae (4 espèces chacune) et Faboideae (2 espèces) mais la densité était plus importante chez les Mimosoideae. Les Anacardiaceae étaient représentées par deux espèces. Les autres familles étaient représentées par une espèce chacune. Selon Ngom et al. (2013), ces familles les plus représentées (Fabaceae, Combretaceae) sont caractéristiques de la végétation ligneuse dans ces zones.

Le fait que les champs contenaient le plus grand nombre d'individus de grande taille s'expliquait par la plus grande surface terrière. En effet, dans ces entités étaient représentées le mieux les populations d'Adansonia digitata, de Cordyla pinnata et de Ficus sur. Ce sont des espèces aux usages multiples et aux rôles importants dans la zone (Sarr et al., 2013a), et elles bénéficient d'une certaine protection de la part des propriétaires de champs (Ngom, 2001 ; 2008). D'autre part, ces champs sont des parcs agroforestiers et les agriculteurs coupent volontairement des arbres pendant le processus de débroussaillement (Bounkoungou et al., 1993). La grande présence de catégories de diamètre et de hauteur au niveau des champs indique qu'il s'y trouve une plus grande couverture aérienne. Cela s'explique par le fait qu'au niveau des champs ces grands arbres à cime étalée plus ou moins ouverte sont le plus souvent plus adaptés aux conditions climatiques et pédologiques rigoureuses du Sahel (Konaté, 1999). Ceci est renforcé par la création d'un comité de gestion qui surveille les champs. Au niveau de la forêt cela s'explique par sa mise en défens depuis cinq ans. La distance moyenne entre les individus était relativement élevée dans les deux zones ; ce qui pouvait traduire une dégradation du milieu (Sarr et al., 2013b) malgré leur protection. En effet cela s'est traduit par un rapport entre la densité théorique et la densité réelle supérieur à un dans les champs et proche de un dans la forêt, suggérant une distribution en agrégats de la végétation (Diatta et al., 2009), c'est-à-dire présentant des surfaces où les arbres sont regroupés en bosquets et parfois des espaces clairsemés (Akpo, 1998).

Le peuplement ligneux à différents niveaux de développement était très jeune. En fait, la plupart des individus étaient regroupés dans la première catégorie de diamètre et de hauteur. Cela prouve que la régénération était bonne (Akpo, 1998). La valeur de régénération élevée, $98,4 \%$ au niveau de la forêt et $76,34 \%$ au niveau des champs, montrait l'importance de la strate arbustive du peuplement. Les espèces de Combretaceae se régénéraient le mieux. Dans tous les types d'exploitations étudiés, le taux de régénération de Combretum glutinosum et Guiera senegalensis ont dépassé $80 \%$. Cela reflétait en outre leur grande capacité à coloniser des milieux anthropisés. Toutes les autres espèces à l'exception de Piliostigma reticulatum et de Ozoroa insignis, avaient un indice spécifique de régénération très faible. Si l'on sait que dans la plupart des cas, leurs graines sont transportées et vendues dans des centres urbains ou destinées à la consommation, alors pour Adansonia digitata et Cordyla pinnata on comprend que leurs rejets soient absents ou rares (Sarr et al., 2013 ; Bakhoum, 2013 ; Sarr, 2009).

Pendant la saison sèche, les plantes ligneuses jouent un rôle particulier dans l'alimentation des animaux car, selon Kaboré (1995), plus de $75 \%$ des arbres et arbustes en Afrique de l'Ouest sont fourragers. Parmi les $82 \%$ espèces inventoriées comme fourragères, $44 \%$ sont très appétées, $17 \%$ sont appétées et $39 \%$ sont peu appétées. Ces résultats confirment ceux obtenus par Savadogo (2002) pour les espèces inventoriées en commun (respectivement $57 \%, 14 \%$ et $29 \%$ ) dans le terroir de Tiogo en zone soudanienne. Ils sont toutefois contraires à ceux de Le Houerou (1980) qui souligne l'importance numérique des espèces peu appétées dans la même zone climatique. Cela peut s'expliquer par les variations intrazones en offre d'espèces ligneuses fourragères, reflet des conditions édaphiques et des variations pluviométriques (Boudet, 1984).

Dans la quête de fourrage ligneux, les bovins, les ovins et les caprins sont conduits séparément au pâturage du fait de leurs exigences alimentaires (Daget et Godron, 1995). D'après Petit (2000), les agropasteurs secouent au moyen de gaules ou bâtons les branches des ligneux afin de mettre à la disposition des bovins les fruits inaccessibles dès que la hauteur du ligneux est supérieure à deux mètres. Le même constat est fait par Hoffmann (1985) ; les petits ruminants sont conduits par les enfants à proximité des champs. Ces enfants pratiquent l'émondage des ligneux fourragers au profit des petits 
ruminants lorsqu'il y a rupture des stocks des résidus de récolte et raréfaction des ressources herbacées. Les feuilles avec les fruits et les rameaux constituent les parties les plus utilisées par les ruminants.

\section{CONCLUSION}

Dans le cadre de la gestion et de la conservation des ressources dans la région de Kaffrine, cette étude fournit plus d'informations sur l'état actuel des peuplements ligneux dans la forêt et dans les champs de Ngouye. Elle a permis de caractériser la végétation dans ces deux unités de production. L'évaluation de la diversité floristique a révélé l'existence de 28 espèces réparties entre 25 genres et 15 familles. Les Combretaceae étaient les mieux représentées, suivies des sous-familles Caesalpinioideae et des Mimosoideae.

L'étude a également permis de corroborer l'idée selon laquelle la strate ligneuse était à dominance arbustive. L'état actuel de la régénération était fortement perturbé (espèces vieillissantes, manque de jachère...) pour la plupart des espèces citées (plus de $80 \%$ présentaient une régénération rare ou absente).

En outre, les ligneux assurent des fonctions diverses et jouent un rôle social très important. Ils interviennent aussi dans l'alimentation du bétail. Face au contexte actuel de désertification et de changement climatique, cette étude a révélé la nécessité d'apporter des mesures politiques efficientes et opportunes de conservation et de rétablissement de certaines espèces ligneuses en favorisant surtout la population autochtone. En guise de perspectives, il serait intéressant de mener d'autres études comme la détermination de la composition chimique des feuilles des arbres les plus utilisés ce qui approfondira davantage les connaissances sur la qualité des fourrages consommés. La promotion de la régénération naturelle assistée, le développement des cultures fourragères (rendant les agriculteurs sensibles aux avantages de ces cultures) et une taille modérée (par exemple, limitée à un quart de la canopée fourragère) peuvent aider à réduire la pression sur les ligneux.

\section{Conflits d'intérêts}

Létude a été réalisée sans conflit d'intérêts.

\section{Déclaration des contributions des auteurs}

$\mathrm{AbD}$ et $\mathrm{SN}$ ont participé à la conception et à la planification de l'étude ; $\mathrm{AbD}$ a recueilli les données et rédigé la première version du manuscrit ; OS a participé à la planification de l'étude ; AbD et AlD ont effectué les analyses statistiques; $\mathrm{AG}$ et $\mathrm{SN}$ ont révisé le manuscrit.

\section{REFERENCES}

Agence canadienne de développement international (ACDI), 1985. Une solution à long terme pour le Sahel: I'assistance bilatérale canadienne, Québec, 28 p.

Akpo L.E., Gouzis M., 1996. Influence du couvert sur la régénération de quelques espèces ligneuses sahéliennes (Nord Sénégal, Afrique occidentale). Webbia., 50 (2): 247-263, doi: 10.1080/00837792.1996.10670605

Akpo L.E., 1998. Effets de l'arbre sur la végétation herbacée dans quelques phytocénoses du Sénégal : variation selon un gradient climatique. Thèse Doct. d'Etat en Sciences naturelles, Université Cheikh Anta Diop de Dakar (UCAD), Dakar, Sénégal, 142 p.

Agence Nationale de la Statistique et de la Démographie (ANSD), 2013. Service régional de la Statistique et de la Démographie, Kaffrine, Sénégal

Agence Nationale de la Statistique et de la Démographie (ANSD), 2013. Situation économique et sociale régionale, Kaffrine, Sénégal, 16 p.

Bakhoum A., 2013. Dynamique des ressources fourragères : indicateur de résilience des parcours communautaires de Téssékéré au Ferlo. Thèse Doct. Université Cheikh Anta Diop de Dakar (UCAD), Dakar, Sénégal, 101 p.
Berhaut J., 1967. Flore du Sénégal. Editions Clair Afrique, Dakar, Sénégal, $485 \mathrm{p}$.

Bognounou O., Ganaba S., Ouadba J.M., 2004. Plantes de construction d'habitations en région sahélienne (Burkina Faso). Bois For. Trop., 282 (4): 11-17, doi: 10.19182/bft2004.282.a20215

Boudet G., 1984. Manuel sur les pâturages tropicaux et les cultures fourragères. Ministère de la Coopération. IEMVT, Paris, France, 267 p.

Bounkoungou E.G., Ayuk A.T., Zoungrana I., 1993. Les parcs agro-forestiers des zones semi-arides d'Afrique de l'Ouest. Symposium international, Ouagadougou, Burkina Faso, 226 p.

Breman H., Kessler J.J., 1995. Woody plants in agro-ecosystems of semi-arid regions (an emphasis on the Sahelian countries), Adv. Ser. Agric. Sci., 23: 340 p., doi : 10.1007/978-3-642-79207-6

Daget P.H., Godron M., 1995. Pastoralisme : Troupeaux, espaces et sociétés. HATIER, AUPELF, UREF, Universités francophones, Paris, France, 510 p.

Diatta C.D., Gueye M., Koma S., Akpo L.E. 2009. Diversité de la flore et de la végétation de la réserve de Ngazobil (Joal-Fadiouth) au Sénégal. J. Sci., 9 (3): $1-13$

Fall S.T., Traoré E.H., Cissé M., Korea A., Sèye B.M., Fall A., Diop A.K., 1994. Système d'alimentation pour la production intensive de viande au Sénégal. Essai de prévulgarisation de rations d'embouche. Convention ISRA-CRDI, Dakar, Sénégal, 38 p.

Fall T., Traoré E., Ndiaye K., Ndiaye N.S., Sèye B.M., 1997. Utilisation des fruits de Faidherbia albida pour l'alimentation des bovins d'embouche paysanne dans le bassin arachidier au Sénégal. Livest. Res. Rural Dev. 9 (5): $17 \mathrm{p}$.

FAO, 1999. L'insécurité alimentaire dans le monde : la faim au quotidien et la crainte permanente de la famine. Rome, Italie, $40 \mathrm{p}$.

FAO, 2003. Situation des forêts du monde 2003. Rome, Italie, $12 p$

Gounot M., 1969. Méthodes d'études quantitatives de la végétation. Masson, Paris, France, 314 p.

Groupe d'experts intergouvernemental sur l'évolution du climat (GIEC), 2007. Changement climatique 2007, Genève, Suisse, 114 p.

Guerin H., 1984. Lexique 'Latin Wolof ' des espèces végétales consommées par les ruminants domestiques à Thyssé-Kaynor-Sonkorong. LNERV/ISRA, Dakar, Sénégal, 6 p.

Hoffmann O., 1985. Pratiques pastorales et dynamique du couvert végétal en pays Lobi. Nord-est de la Côte d'Ivoire, Orstom, Paris, France, 355 p.

Kaboré Z., 1995. Composition chimique et valeur nutritive des herbacées et ligneux des pâturages naturels soudaniens et des sous-produits du Burkina Faso. Thèse d'Etat, Université Ouagadougou, Burkina Faso, 201 p.

Kiema S., 2007. Elevage extensif et conservation de la diversité biologique dans les aires protégées de l'Ouest burkinabé. Arrêt sur leur histoire, épreuves de la gestion actuelle, état et dynamique de la végétation. Thèse Doct., Université d'Orléans, France, 658 p.

Konaté P.S., 1999. Structure, composition et distribution de quelques ligneux dans les provinces du Seno et du Yagha: proposition d'application à leur gestion. Mém. fin d'études d'ingénieur du développement rural, Université Polytechnique de Bobo-Dioulasso, Burkina Faso, 76 p.

Le Houérou H.N., 1980. Rôle des ligneux fourragers dans les zones sahélienne et soudanienne. In le Houérou H.N. ed., Collogue international sur les fourrages ligneux en Afrique. Addis Abeba, Ethiopie, 08-12 Août 1980, CIPEA, 85-101.

Mohamed Mahamoud C., Lo M., Bassène E., Akpo L.E., 2008. Caractéristiques de la flore et végétation ligneuses de forêts communautaires de la zone soudano-sahélienne au Sénégal. J. Sci. Technol., 6 (2): 72- 85

Ngom D., 2001. Place de l'arbre dans les systèmes de production de la NEMA dans le NIOMBATO (Saloum, Sénégal), Mémoire DESS CRESA, Niamey, Niger, 69 p.

Ngom D., 2008. Définition d'indicateurs de gestion durable des ressources sylvopastorales au Ferlo. (Nord-Sénégal). Thèse Doct., Université Cheikh Anta Diop de Dakar (UCAD), Dakar, Sénégal, 148p.

Ngom D., Fall T., Sarr O., Diatta S., Akpo L.E., 2013. Caractéristiques écologiques du peuplement ligneux de la réserve de biosphère du Ferlo (Nord Sénégal). J. Appl. Biosci., 65 (2013): 5008-5023, doi: 10.4314/jab. v65i0.89644 
Petit S., 2000. Environnement des troupeaux et usage de l'arbre chez les agropasteurs peuls de l'Ouest burkinabé. Approche comparative et systématique de trois situations : Barani, Kourouma et Ouangolodougou. Thèse Doct., Université d'Orléans, France, 528 p.

Poupon H., 1980. Structure et dynamique de la strate ligneuse d'une steppe sahélienne au nord du Sénégal. ORSTOM ed. (Etudes \& Thèses), Paris, France, 307 p.

République du Sénégal, 2006. Ministère de l'Économie et des Finances (MEF) : Doc. stratégie de réduction de la pauvreté II : Dakar, Sénégal

Roberts-Pichette P., Gillespie L., 2002. Protocoles de suivi de la biodiversité végétale terrestre. Collection des publications hors-série du RESE, rapport 9. Centre canadien des eaux intérieures, Ontario, Canada, 138 p.

Rondeux J., 1993. La mesure des arbres et des peuplements forestiers. Presses agronomiques, Gembloux, Belgique, 521 p.

Sarr O., 2009. Caractéristiques des ligneux fourragers dans les parcours communautaires de Lour Escale (région de Kaffrine centre-Sénégal). DEA, FST, UCAD, Dakar, Sénégal, 61 p.

Sarr O., Bakhoum A., Diatta S., Akpo LE, 2013a. L'arbre en milieu soudano-sahélien dans le basin arachidier (Centre-Sénégal). J. Appl. Biosci., 61 (2013): 4515-4529, doi: 10.4314/jab.v61i0.85598
Sarr O., Ngom D., Bakhoum A., Akpo L.E., 2013b. Dynamique du peuplement ligneux dans un parcours agrosylvopastoral du Sénégal. Vertigo, 13 (2), doi : 10.4000/vertigo.14067

Sarr O., Diatta S., Gueye M., Ndiaye P.M., Guissé A., Akpo L.E., 2013c. Importance des ligneux fourragers dans un système agropastoral au Sénégal (Afrique de l'ouest). Rev. Méd. Vét., 164 (1): 2-8, doi: hal-01722601

Sarr O., Ngom D., Ngarya F.T., Diatta S., Akpo L.E., 2014. Etat de la végétation ligneuse dans trois unités d'utilisation des terres d'une zone agropastorale au Sénégal (région de Kaffrine). Int. J. Biol. Chem. Sci., 8 (6): 26692684, doi: 10.4314/ijbcs.v8i6

Savadogo P., 2002. Pâturages de la forêt classée de Tiogo : Diversité végétale, productivité, valeur nutritive et utilisations. Mém. Ingénieur, Université Polytechnique de Bobo Dioulasso, Burkina Faso, 105 p.

Service régional de la Statistique et de la Démographie (SRSD), 2013. Situation économique et sociale régionale, Kaffrine, Sénégal

Veillard P., 2011. L'avenir de l'élevage africain. Défis sud. SOS FAIM, Bruxelles, Belgique, 11-13

Zoungrana I., 1991. Recherche sur les aires pâturées du Burkina Faso : Aménagement et ressources naturelles. Thèse d'Etat, Université Bordeaux III France, $277 \mathrm{p}$

\section{Summary}

Dione A., Ngom S., Sarr O., Diallo A., Guissé A. Characteristics of the woody stands of two land-use systems in Kaffrine Region, Senegal

The study focused on the characterization of the woody stands of two land-use systems (a forest closed to grazing animals, and fields) in the Ngouye terroir in Senegal. It was carried out in an agropastoral area that hosts several families of transhumants annually. By means of woody vegetation sampling and surveys of the population we inventoried 28 species belonging to 25 genera and 15 botanical families. The Fabaceae (subfamilies: Mimosoideae, Caesalpinioideae, Faboideae) were the most represented, followed by the Combretaceae. The parameters of the study showed that there were clear differences between the two systems, which highlighted their heterogeneity, with a great stability of the stands in the forest. The stand was generally very young, with a large percentage of individuals in the first diameter-and-height category. The fields had the highest proportion of mature trees but the regeneration rate in this area was lower than in the forest. The species richness of the fields was much higher than that of the forest. In terms of pastoral activities, woody plants were used to feed livestock.

Keywords: woody plants, feed resources, agropastoral systems, forest stands, Senegal

\section{Resumen}

Dione A., Ngom S., Sarr O., Diallo A., Guissé A. Características del asentamiento leñoso de dos sistemas de uso de la tierra en la región de Kaffrine en Senegal

El estudio se centró en la caracterización del asentamiento leñoso de dos sistemas de uso de la tierra (el bosque como zona de exclusión del pastoreo) en el territorio de Ngouye en Senegal. Se llevó a cabo en una zona agro pastoral que acoge anualmente a varias familias de trashumantes. Mediante muestreos de vegetación leñosa y encuestas a la población, identificamos 28 especies pertenecientes a 25 géneros y 15 familias botánicas. Las Fabaceae (subfamilias: Mimosoideae, Caesalpinioideae, Faboideae) fueron las más representadas, seguidas de las Combretaceae. Los parámetros del estudio mostraron la existencia de diferencias claras entre las dos unidades, mostrando su heterogeneidad, con una gran estabilidad de los asentamientos en el bosque. Los rodales leñosos eran generalmente muy jóvenes, con un gran porcentaje de individuos en la primera categoría de diámetro y altura. Los campos presentaban la mayor proporción de individuos adultos, pero la tasa de regeneración en esta zona fue menor que la del bosque. La riqueza específica de los campos fue mucho mayor que la del bosque. A nivel pastoral, las leñosas participaron en la alimentación del ganado.

Palabras clave: plantas leñosas, recursos de piensos, sistemas agropascícolas, rodales, Senegal 
